\title{
Estimation of brain connectivity through Artificial Neural Networks
}

\author{
Yuri Antonacci, Member, IEEE, Jlenia Toppi, Member, IEEE, Donatella Mattia, Antonio Pietrabissa \\ and Laura Astolfi, Member, IEEE
}

\begin{abstract}
Among different methods available for estimating brain connectivity from electroencephalographic signals (EEG), those based on MVAR models have proved to be flexible and accurate. They rely on the solution of linear equations that can be pursued through artificial neural networks (ANNs) used as MVAR model. However, when few data samples are available, there is a lack of accuracy in estimating MVAR parameters due to the collinearity between regressors. Moreover, the assessment procedure is also affected by the lack of data points. The mathematical solution to these problems is represented by penalized regression methods based on $l_{1}$ norm, that can reduce collinearity by means of variable selection process. However, the direct application of $l_{1}$ norm during the training of an ANN does not result in an efficient learning. With the introduction of the stochastic gradient descent-L1 (SGD-L1) it is possible to apply $l_{1}$ norm directly on the estimated weights in an efficient way. Even if ANNs has been used as MVAR model for brain connectivity estimation, the use of SGD-L1 algorithm has never been tested to this purpose when few data samples are available. In this work, we tested an approach based on ANNs and SGD-L1 on both surrogate and real EEG data. Our results show that ANNs can provide accurate brain connectivity estimation if trained with SGD-L1 algorithm even when few data samples are available.
\end{abstract}

\section{INTRODUCTION}

Brain connectivity is widely used for better understanding the interactions between brain regions, and the influences that neural structures exert to one another. Among different methods for estimating brain connectivity from electroencephalographic signals (EEG), those based on the use of multivariate autoregressive models (MVAR) are the most widely used. The classical form of these models represents the interactions between brain regions in the form of linear equations [1]. The solution can be pursued through different methods, among which artificial neural networks (ANNs). In the literature, ANNs proved to be a very effective tool for linear time series analysis in different contexts [2]. They can rely on a training process, through an optimization algorithm, for performing linear regression. Independently from the used methods, a direct relationship between the error in estimating MVAR parameters and the decrease of data samples available has been showed [3]. In particular, to ensure the accuracy of the estimation process, the ratio between the number of data samples and the number of

\footnotetext{
*Research partially supported by the European Union's Horizon 2020 program under the Marie Skłodowska-Curie grant agreement No 778234 (DOCMA), by Sapienza University of Rome - Progetti di Ateneo 2016 (MIME-BCI- PI1161550696379A), Progetti di Ateneo 2017 (EMBRACING- RM11715C82606455) and Progetti di avvio alla ricercar 2018

Y. Antonacci, J. Toppi, and L. Astolfi are with the Department of Computer, Control, and Management Engineering, University of Rome
}

MVAR parameters to be estimated should be greater than 10 . When this is not the case, the estimation process become very challenging due to the high correlation between regressors [4]. Moreover, during a brain connectivity analysis, a statistical assessment is needed to discard spurious links. Unfortunately, also the performances of this step are affected by the number of data samples available [5]. A mathematical solution to this problem is represented by penalized regression methods. Among these, those based on $1_{1}$-norm involving a penalty term in the cost function to minimize (usually MSE function [6]). This constraint leads to a reduction of collinearity with the selection of a subset of MVAR parameters from the whole set of parameters. This procedure reduces: i) the mean squared error related with the estimation process and ii) the error in discarding spurious links during the assessment procedure. In literature, the penalized regression techniques have proved their effectiveness in EEG and fMRI context [7], [8]. However, in the framework of ANNs, the direct application of $1_{1}$ norm during the learning process does not result in an efficient training. Only recently a new training algorithm, named stochastic gradient descent-L1 [9] (SGD-L1), was introduced in the literature, allowing to apply $1_{1}$-norm during the training process directly on the estimated weights with the result of an efficient training process. The use of ANNs as MVAR model for the brain connectivity estimation has been proposed in [10]. However, the use of SGD-L1 algorithm has never been tested for the purposes of reducing collinearity in the estimation of MVAR parameters and performing the assessment of estimated connectivity patterns. In this work, we propose an approach for the estimation of brain connectivity based on ANNs, with the aim to provide an accurate estimate of MVAR parameters and assessment procedure also in the case of short data trials. The methodology was tested: (i) in different conditions of data samples available on EEG simulated data; (ii) with real EEG data during a motor imagery task performed by a healthy subject.

\section{METHODS}

\section{A. Multivariate Autoregressive model (MVAR)}

For a time series $\boldsymbol{x}(n)=\left[x_{1}(n), x_{2}(n), \ldots, x_{M}(n)\right]^{T}$, an MVAR model can be defined as:

Sapienza, Rome, Italy, and with IRCCS Fondazione Santa Lucia, Rome, Italy.

D. Mattia is with IRCCS Fondazione Santa Lucia, Rome, Italy.

A. Pietrabissa is with the Department of Computer, Control, and Management Engineering, University of Rome Sapienza, Rome, Italy. 


$$
\left[\begin{array}{c}
x_{1}(n) \\
\vdots \\
x_{M}(n)
\end{array}\right]=\sum_{k=1}^{p} \boldsymbol{A}_{k}\left[\begin{array}{c}
x_{1}(n-k) \\
\vdots \\
x_{M}(n-k)
\end{array}\right]+\left[\begin{array}{c}
e_{1}(n) \\
\vdots \\
e_{M}(n)
\end{array}\right]
$$

The matrix $\boldsymbol{A}_{k}$ is given by:

$$
\boldsymbol{A}_{k}=\left[\begin{array}{ccc}
a_{11}^{k} & \ldots & a_{1 M}^{k} \\
\vdots & \ddots & \vdots \\
a_{M 1}^{k} & \ldots & a_{M M}^{k}
\end{array}\right], k=1,2, \ldots, p
$$

where $\mathrm{p}$ is the model order and the vector $\mathbf{E}$ is the innovation process assumed to be white and uncorrelated. Independence between a pair of signals result in zero coefficients while dependence is reflected in nonzero values [10].

\section{B. ANN as a multivariate autoregressive model}

To the purposes of this study, the neural network inputs are the lagged samples of the $\mathrm{M}$ time series at different delays. During the training, the network tries to predict the next samples (output), on the basis on the previous samples in input. A schematic representation of this procedure is shown in Figure 1. After training the network, the connectivity relationships are represented by the weights of the network.

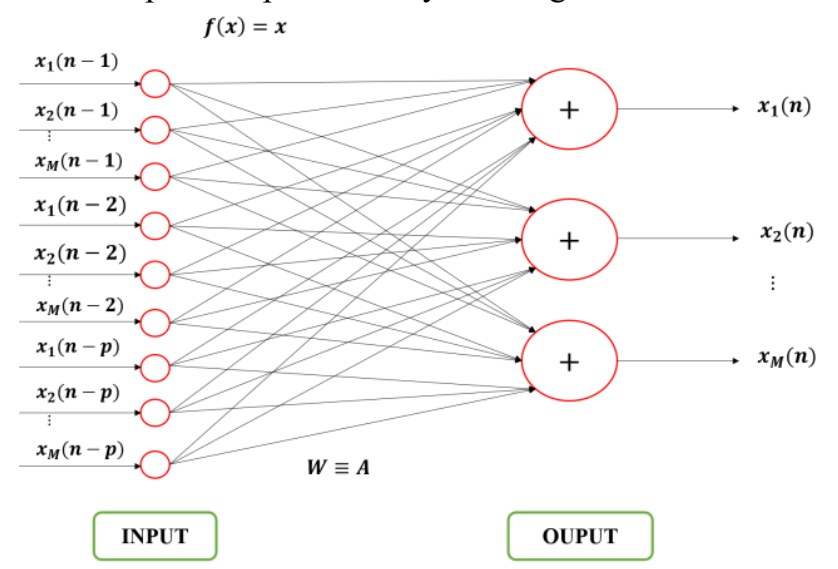

Figure 1. schematic representation of the ANN used as MVAR model.

\section{Training of the network}

The weights of the network are randomly initialized. The network is trained using the SGD-L1 algorithm that includes two steps. Firstly, the weights are updated without considering the $l_{1}$ term. Then, the $l_{1}$ norm is applied to the weights. At the end of the training process, many of the weights in the network become zero. The number of zeroes is controlled by a penalty parameter selected during the training process. Specifically, the MSE function is minimized for each penalty parameter by means of Generalized Cross Validation (GCV) method [11] (50\% training, 25\% validation and 25\% testing randomly selected). We run the optimization algorithm for 500 epochs with full batch size and a learning rate equal to 0.001 .

\section{SIMULATION STUDY}

The simulation study consisted of the following steps:

i. Generation of simulated EEG-like datasets, fitting predefined ground truth network of 10 nodes, under different conditions of $\mathrm{K}$ ratio (factor $\mathrm{K}$ : $0.5,1,3,5,10$ ). $\mathrm{K}$ is defined as the number of data samples available for the training of the ANNs divided by the number of MVAR coefficients to be estimated. The simulated data were generated as described in [12]. The structure of the network varied along the iterations. The density was set at $25 \%$.

ii. Definition and training of the network for the selection of the optimal penalty parameter by means of GCV method.

iii. Estimation of the MVAR parameters by means of a NN with the selected penalty parameter.

iv. Evaluation of the accuracy of ANNs in the estimation of no-null connection. We used the Mean Absolute Percentage Error (MAPE) [13]. Let $A$ and $\hat{A}$ denote the vector of imposed and estimated values of MVAR parameters. the MAPE is defined as follows:

$$
\text { MAPE }=\frac{1}{N} \sum_{n=1}^{N}\left|\frac{\boldsymbol{A}_{n}-\widehat{\boldsymbol{A}}_{n}}{\boldsymbol{A}_{n}}\right|
$$

where $\mathrm{N}$ is the number of non-null connections. MAPE can range from $0 \%$ to any value.

v. Evaluation of the performances of ANNs in the assessment, through false positive and false negative rates, summarized by ROC curve and the related Area Under Curve (AUC) [14]. AUC values range between 0.5 (random assessment procedure) and 1 (correct assessment procedure).

The entire procedure was repeated 100 times. The two indices (MAPE and AUC) were subjected to a one-way repeated measures ANOVA considering as within factor $\mathrm{K}$ for both MAPE and AUC.

\section{Application to REAl EEG Data}

The real EEG data used for testing ANNs for the estimation of brain connectivity were recorded in a healthy volunteer (male, under 30 and right-handed) performing a motor imagery task (MI). The task consisted of 90 trial of right hand MI and 90 trials of foot MI. Signals were recorded by a 45channels EEG system (electrodes located according to the 1020 International System). Raw EEG data and further details about the performed pre-processing can be found in [15].

\section{A. Single trial connectivity estimation}

Firstly, we selected 11 out of the 45 EEG channels (C5, C3, $\mathrm{C} 1, \mathrm{C} 2, \mathrm{C} 4, \mathrm{C} 6, \mathrm{CP} 3, \mathrm{CP} 4, \mathrm{Cz}, \mathrm{CPz}, \mathrm{FCz}$ ) in order to match the dimension of the networks explored in the simulation study. Then we performed the following steps:

i. Segmentation of the EEG signals, selecting 100 samples between the third and the fourth second [15].

ii. Estimation of the model order for each condition by means of final prediction error, as suggested in [15] $(\mathrm{K}=1)$.

iii. For each trial, definition and training of an ANN for the selection of the optimal penalty parameter through GCV ( $80 \%$ training, 10\% validation and $10 \%$ testing).

iv. MVAR parameters estimation through the training of an ANN with the penalty parameter previously selected (for each trial).

v. Fourier Transform of the MVAR parameters to obtain the transfer functions $\mathrm{A}_{\mathrm{ij}}(\mathrm{f})$.

\section{B. Classification task}

To test the accuracy of the connectivity estimation performed by ANNs, we tested the capability of the networks to characterize the two tasks (hand MI and foot MI) by means of a classification study. For each condition and for each trial the 
$\mathrm{A}_{\mathrm{ij}}(\mathrm{f})$ were averaged within two different frequency bands: alpha $(8-12 \mathrm{~Hz})$ and beta $(13-30 \mathrm{~Hz})$ and used as features for the classification procedure. A sub-selection of the features was made by selecting the $25^{\text {th }}$ highest ranked one according to the associated t-value (independent sample t-test, Hand vs Foot) [16].

A feed forward neural network (FFNN) was used as a classifier. This includes an input layer, a hidden layer with one neuron (sigmoid activation function) and an output layer with a soft-max activation function. The data were split in $70 \%$ for training process (126 trials out of 180 ), $15 \%$ for validation and $15 \%$ for testing. Networks training was performed using Gradient Descent algorithm with a learning rate equal to 0.001 , whereas a cross-entropy function was minimized during the training process. Training phase was stopped by the early stopping criterion [17]. The process was repeated 100 times, with a randomization of the observations, for each frequency band, in order to verify if the classification performances were significantly higher than the chance level. As a measure of the performances, we computed the classification accuracy (ACC) on the test set at each iteration and for each frequency band. ACC index was subjected to a one-way repeated measures ANOVA considering as within factor the frequency band analyzed (factor BAND).

$$
\text { V. RESULTS }
$$

\section{A. Simulation study}

Table 1 reports the ANOVA results for MAPE and AUC parameters and shows a significant effect of the $\mathrm{K}$ factor on both MAPE and AUC. Figure 2a shows the plot of means for MAPE. The panel highlights the decrease of MAPE with the increases of the $\mathrm{K}$ ratio. As expected, there is a decreasing of the error in estimating the value of MVAR parameters when the number of data samples increases.

Moreover, in the condition of high collinearity $(\mathrm{K}=0.5)$ MAPE assumes a mean value around $50 \%$. Instead, when the number of data samples increases $(K=10)$, the average value of MAPE is around $20 \%$.

TABLE I. ANOVA RESULTS FOR MAPE AND AUC INDEXES

\begin{tabular}{|c|c|}
\hline \multirow{2}{*}{ Parameters } & ANOVA within factors \\
\cline { 2 - 2 } & $\boldsymbol{K}$ \\
\hline MAPE & $946,78 * *$ \\
\hline AUC & $213,97 * *$ \\
\hline DoF & $(4,396)$ \\
\hline
\end{tabular}

Results of the one-way ANOVA (F values) on MAPE and AUC parameters. Symbol ** means $\mathrm{p}<0.0001$

Figure $2 \mathrm{~b}$ shows the plot of means for AUC. In particular, there is an increase of the AUC with the increase of the K ratio, which means with the increasing of the data samples available for the estimation process. The accuracy in discarding spurious links seems to be very high even when there is high collinearity. In fact, when $\mathrm{K}=0.5$ the average value of AUC is around $85 \%$. The situation is very different for $\mathrm{K}$ equal to 10 because the mean value of $\mathrm{AUC}$ is close to 1 (meaning correct classification). a)

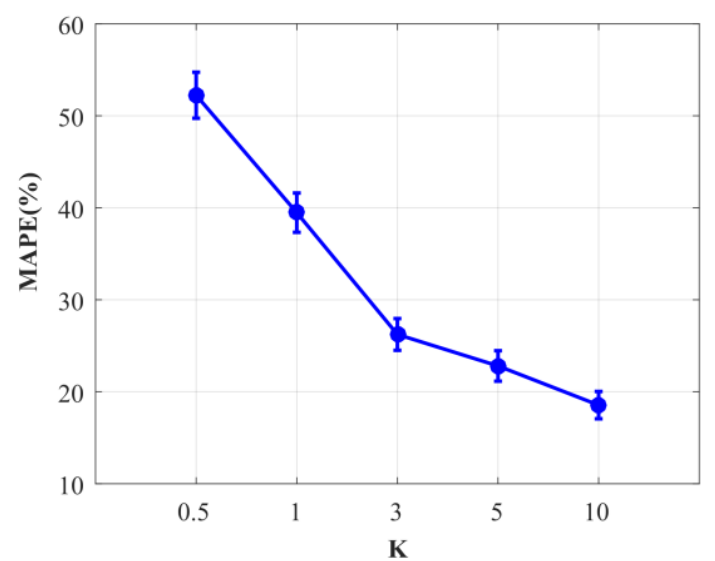

b)

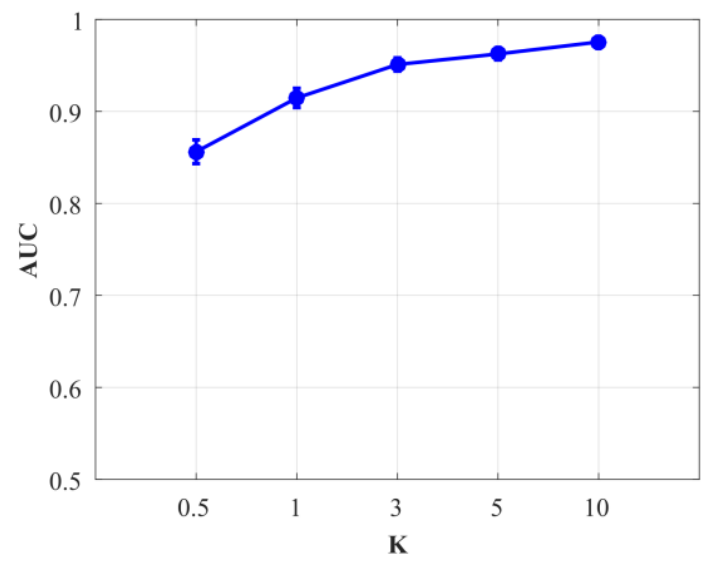

Figure 2- Plot of means of MAPE and AUC (panel a and b) indices. The diagram shows the mean values of the two indices for different values of K. The bars represent $95 \%$ confidence interval

\section{B. Application to real EEG data}

In figure 3 we reported the boxplot describing the results of the one-way ANOVA performed on classification accuracy, considering as within main factor the frequency band analysed. The median value for classification accuracy (black line in the middle of the boxes) in all the conditions analysed shows a value above the chance level (0.5, random classification).

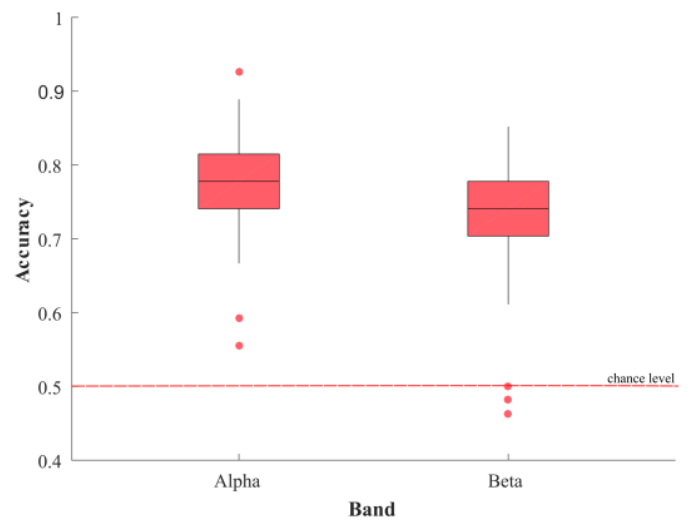

Figure 3. Statistical boxplot reporting results of one-way ANOVA $\left(\mathrm{F}(1,99)=16,1 ; \mathrm{p}<10^{-3}\right)$ computed considering as dependent variable the classification accuracy (ACC) and as within main factor BAND 
The median value of classification accuracy in the alpha band is nearly 0.8 , while in the beta band it assumes a value slightly higher than 0.7 . It can be noticed how in the distribution of classification accuracy values for the beta band, there are at least three outliers below the chance level. Instead, in the alpha band all the classification accuracy values associated with the 100 iterations are above the chance level.

\section{DISCUSSION}

Results of the simulation study shows how it is possible to provide and accurate estimation of brain connectivity by means of ANNs in different conditions of data samples available. In particular, the combined use of ANNs as MVAR models and the SGD-L1 algorithm for the training process provides a solution on both estimation of MVAR parameters and assessment of the estimated patterns. This is possible thanks to the regularization applied to the weights during the training process.

MAPE showed a decreasing trend with the increase of the number of data samples available for the estimation process. This aspect has already been highlighted in previous works in the context of linear regression analysis. In [3] they showed how the decreasing of the number of data samples available for the estimation corresponds to a decrease of performance in term of Mean Squared Error. More in general, in the context of time series analysis, the reduction of the number of data samples is related to a collinearity between regressors and a consequent decreases in performance [4]. From the point of view of the assessment procedure, figure $2 \mathrm{~b}$ shows how the performances in discarding spurious links improves as the number of data samples increases, as expected [5]. In the framework of penalized regression techniques, Tibshirani introduced LASSO regression that overcomes the problem related to the effects of collinearity by means of a variable selection technique [6]. These techniques showed outstanding results in the context of brain connectivity estimation from fMRI and EEG data [7], [8]. Other studies showed how to use an ANN as MVAR model, but testing this only for huge amounts of data samples. Furthermore, the problem related with the assessment procedure was not addressed. To our knowledge this is the first time in the EEG context, that an ANN used as MVAR model and trained with SGD-L1 algorithm is proposed and tested for the purposes of brain connectivity estimation when few data samples are available. Figure 3 shows how it is possible to obtain promising classification accuracy of EEG data using features extracted from a brain connectivity analysis, performed by means of ANNs in a condition of collinearity $(\mathrm{K}=1)$. Firstly, the highest value of accuracy is reached in the alpha band. Other works showed how the maximum value of accuracy is associated with the frequency band related to the motor imagery tasks. For example, in [18] the highest value of accuracy, in the discrimination between hand and foot motor imagery tasks, is reached by using the power spectral density in the alpha band as feature. Other studies in the literature used features derived from a brain connectivity analysis for a classification task [19]. Rathee et al showed how it is possible to discriminate between Hand and Foot motor imagery conditions, with values of estimated links between different EEG time series used as features. Although the results of figure 3 were performed on few data samples, they are comparable with those obtained in [19].

Despite the promising results obtained in this study, there are some drawbacks that will need further investigations. In fact, the impact of changing the parameters for ANN training, such as learning rate, batch size and number of epochs, to the brain connectivity estimation is not known. Furthermore, from an applicative point of view it would be interesting to increase the experimental sample in order to confirm the results obtained in a single subject analysis.

In summary, in this work we proposed and tested an approach based on ANNs for the estimation of brain connectivity that provides evidences about its usability even when few data samples are available. The results pave the way to the possible use of this approach for single-trial analysis and for real time analysis, for instance for brain computer interfaces (BCI) applications.

\section{REFERENCES}

[1] L. Astolfi et al., «Comparison of different cortical connectivity estimators for high-resolution EEG recordings», 2007.

[2] M. Paliwa et al., «Neural networks and statistical techniques: A review of applications», Expert systems with applications, 2009.

[3] A. Schlögl, «A comparison of multivariate autoregressive estimators», Signal processing, 2006

[4] M. Irfan et al., «Comparison of Shrinkage Regression Methods for Remedy of Multicollinearity Problem», MEJS, 2013.

[5] Y. Antonacci et al., «Estimating brain connectivity when few data points are available: Perspectives and limitations», in 2017 39th Annual International Conference of the IEEE Engineering in Medicine and Biology Society (EMBC), 2017.

[6] R. Tibshirani, «Regression Shrinkage and Selection via the Lasso», Journal of the Royal Statistical Society. 1996.

[7] P. A. Valdés-Sosa et al., «Estimating brain functional connectivity with sparse multivariate autoregression», 2005.

[8] T. R. Mullen et al., «Real-Time Neuroimaging and Cognitive Monitoring Using Wearable Dry EEG», IEEE TBME, 2015.

[9] Y. Tsuruoka et al., «Stochastic gradient descent training for 11regularized log-linear models with cumulative penalty», in Proceedings of the Joint Conference of the 47th Annual Meeting of the ACL and the 4th International Joint Conference on Natural Language Processing of the AFNLP: Volume 1-Volume 1, 2009.

[10] N. Talebi et al., «Estimation of effective connectivity using multilayer perceptron artificial neural network», Cogn Neurodyn, 2018.

[11] X. Sun, «The Lasso and its Implementation for Neural Networks», 1999.

[12] J. Toppi et al., «Testing the Significance of Connectivity Networks: Comparison of Different Assessing Procedures», IEEE TBME, 2016

[13] S. Kim et al. , "A new metric of absolute percentage error for intermittent demand forecasts», International Journal of Forecasting, 2016.

[14] J. Toppi et al., «Measuring the agreement between brain connectivity networks», in 2016 38th Annual International Conference of the IEEE Engineering in Medicine and Biology Society (EMBC), 2016.

[15] M. Billinger et al., "Single-trial connectivity estimation for classification of motor imagery data», J Neural Eng, 2013.

[16] S. Theodoridis et al., Introduction to pattern recognition: a matlab approach. Academic Press, 2010.

[17] L. Prechelt, "Automatic early stopping using cross validation: quantifying the criteria», Neural networks : the official journal of the International Neural Network Society, 1998.

[18] F. Cincotti et al., «Classification of EEG mental patterns by using two scalp electrodes and Mahalanobis distance-based classifiers», Methods of Information in Medicine, 2002.

[19] D. Rathee et al., «Single-trial effective brain connectivity patterns enhance discriminability of mental imagery tasks», Journal of neural engineering, 2017. 